\title{
STABILITY OF SOLUTIONS OF LINEAR SYSTEMS WITH RETARDED ARGUMENTS
}

\author{
JOHN ABRAMOWICH
}

\begin{abstract}
A criterion is given for the stability of the trivial solution of a linear system of differential equations with variable coefficients and variable delays under the assumptions of continuity and boundedness of the coefficients and continuous differentiability of the delays. The criterion is a condition on the roots of a certain "quasi-polynomial," i.e. a polynomial in a variable and exponentials of that variable. The derivation is based on Lyapunov's direct method.
\end{abstract}

Introduction. The question of the stability of the trivial solution of systems of the form

$$
x^{\prime}(t)=\sum_{l=0}^{m} A_{l}(t) x\left(t-\tau_{l}(t)\right), \quad t>t_{0},
$$

where $x(t)=\left(x_{1}(t), x_{2}(t), \cdots, x_{n}(t)\right), A_{l}(t) \quad(l=0,1, \cdots, m)$ are $n \times n$ matrices, $\tau_{l}(t)\left(\tau_{0}(t) \equiv 0, \tau_{l}(t)>0, t>t_{0}, l=1, \cdots, m\right)$ are retardations, is resolved, in the case that the coefficients and retardations are constant, if the character of the roots of the "quasi-polynomial"

$$
\left|\lambda I-\sum_{l=0}^{m} A_{l} \exp \left(-\lambda \tau_{l}\right)\right|=0
$$

is known. (Here $I$ is the unit $n \times n$ matrix and $|A|$ denotes the determinant of the matrix $A$.) Namely, the trivial solution of (1) is stable if all the roots have negative real parts and unstable if at least one root has a positive real part [1]. In this paper we consider the general case in which the coefficients and retardations in (1) are nonconstant and under certain assumptions obtain sufficient conditions for stability of the trivial solution. These conditions bear a resemblance to the ones in the case of constant coefficients and retardations and are applicable to this case also. The method is based on the use of Lyapunov functionals as developed by N. N. Krasovskiı $[3],[2]$.

Received by the editors May 26, 1969.

AMS 1969 subject classifications. Primary 3475, 3451.

Key words and phrases. Stability, linear differential system, variable coefficients, variable delays, quasi-polynomial, Lyapunov functional. 
1. Summary of results. We first introduce some notation and definitions.

Let $E_{l}$ denote the set consisting of those values of $t-\tau_{l}(t)$ which are less or equal to $t_{0}$ for $t \geqq t_{0}$. The set $E=U_{l} E_{l}$ shall be called the initial set for the system (1).

We denote

$$
\begin{array}{rlrl}
\|x(s)\|_{\tau}= & \sup _{0 \leq s \leq \leq^{\tau} ; 1 \leq i \leq n}\left|x_{i}(s)\right|, & \|x(t)\| & =\sup _{1 \leq i \leq n}\left|x_{i}(t)\right|, \\
\|x(t)\|_{\tau 2} & =\left[\sum_{i=1}^{n} \sum_{l=1}^{m} \int_{t \rightarrow \tau_{l}(t)}^{t} x_{i}^{2}(s) d s\right]^{1 / 2}, & \|x(t)\|_{2}=\left[\sum_{i=1}^{n} x_{i}^{2}(t)\right]^{1 / 2} .
\end{array}
$$

We now make the following assumptions on the system (1):

(a) The elements of the matrices $A_{l}(t), l=0,1, \ldots, m$ are continuous and bounded functions for $t \geqq t_{0}$.

(b) The retardations $\tau_{l}(t)>0, l=1,2, \cdots, m,\left(\tau_{0}(t) \equiv 0\right)$, are continuously differentiable functions of $t$ for $t>t_{0}$ and $0 \leqq \tau_{l}^{\prime}(t) \leqq \alpha<1$.

(c) The sets $E_{l}$ are nonempty.

Let us denote

$$
A_{0}^{*}(t, \lambda)=(2 \lambda-m) I_{n}-\left(A_{0}(t)+\tilde{A}_{0}(t)\right),
$$

where $I_{n}$ is the unit $n \times n$ matrix and $\tilde{A}$ denotes the transpose of the matrix $A$.

Let

(2) $D(\lambda, t)=\left|A_{0}^{*}(t, \lambda)-\sum_{l=1}^{m}\left(1-\tau_{l}^{\prime}(t)\right)^{-1} A_{l}(t) \tilde{A}_{l}(t) \exp \left(-2 \lambda \tau_{l}(t)\right)\right|$.

We now state the main theorem.

THEOREM 1. If for each $t>t_{0}, D(t, \lambda)=0$ has real roots, let $M(t)$ denote the largest of these, then if $M(t) \leqq \gamma<0$, the trivial solution of (1) is asymptotically stable.

We shall prove this theorem in the next section.

REMARK. In the case that the coefficients and the retardations are all constant this provides a simplified criterion for the stability of the trivial solution of the system (1) as we need to know only the real roots of $D(\lambda, t)=0$. The disadvantage is that it does not provide a criterion for the instability of the trivial solution.

Let us take as an example a $2 \times 2$ system with one retarded argument. 


$$
\left(\begin{array}{l}
x_{1}(t) \\
x_{2}(t)
\end{array}\right)^{\prime}=\left(\begin{array}{l}
a_{11}(t) a_{12}(t) \\
a_{21}(t) a_{22}(t)
\end{array}\right)\left(\begin{array}{l}
x_{1}(t) \\
x_{2}(t)
\end{array}\right)+\left(\begin{array}{l}
b_{11}(t) b_{12}(t) \\
b_{21}(t) b_{22}(t)
\end{array}\right)\left(\begin{array}{l}
x_{1}(t-\tau(t)) \\
x_{2}(t-\tau(t))
\end{array}\right),
$$

and setting $\beta=2 \lambda$, we obtain

$$
\beta^{2}-\beta c_{1}+c_{2}-\beta e^{-\beta \tau} d_{1}+e^{-\beta \tau} d_{2}+e^{-2 \beta \tau} d_{3}=D(\beta / 2, t),
$$

where (omitting arguments)

$$
\begin{aligned}
c_{1}= & \left(2+2 a_{22}+2 a_{11}\right), \\
c_{2}= & \left(1+2 a_{11}\right)\left(1+2 a_{22}\right)-\left(a_{12}+a_{21}\right)^{2}, \\
\left(1-\tau^{\prime}\right) d_{1}= & b_{11}^{2}+b_{12}^{2}+b_{21}^{2}+b_{22}^{2}, \\
\left(1-\tau^{\prime}\right) d_{2}= & \left(b_{11}^{2}+b_{12}^{2}\right)\left(1+2 a_{22}\right)+\left(b_{21}^{2}+b_{22}^{2}\right)\left(1+2 a_{11}\right) \\
& +2\left(a_{12}+a_{21}\right)\left(b_{11} b_{21}+b_{22} b_{12}\right), \\
\left(1-\tau^{\prime}\right)^{2} d_{3}= & \left(b_{11} b_{22}-b_{12} b_{21}\right)^{2} .
\end{aligned}
$$

For stability the roots of $D(\beta / 2, t)=0$ must be negative for all $t$. In general, one would have to use some method of successive approximations to compute the roots, e.g. Newton's method. Let us take the example

$$
\begin{aligned}
\left(\begin{array}{l}
x_{1}^{\prime}(t) \\
x_{2}^{\prime}(t)
\end{array}\right)= & \left(\begin{array}{ll}
-2 & a(t) \\
-a(t) & -3
\end{array}\right)\left(\begin{array}{l}
x_{1}(t) \\
x_{2}(t)
\end{array}\right) \\
& +\left(\begin{array}{l}
b_{11}(t) b_{12}(t) \\
b_{21}(t) b_{22}(t)
\end{array}\right)\left(\begin{array}{l}
x_{1}(t-\log t) \\
x_{2}(t-\log t)
\end{array}\right), \quad t>2,
\end{aligned}
$$

where $a(t), b_{i j}(t)$ are continuous functions of $t$. We have,

$$
D(\beta / 2, t)=\beta^{2}+8 \beta+15+g(t, \beta),
$$

where

$$
\begin{aligned}
g(t, \beta)= & -\frac{\beta t}{t^{\beta}(t-1)}\left(b_{11}^{2}+b_{12}^{2}+b_{21}^{2}+b_{22}^{2}\right) \\
& -\frac{t}{t^{\beta}(t-1)}\left[5\left(b_{11}^{2}+b_{12}^{2}\right)+3\left(b_{21}^{2}+b_{22}^{2}\right)\right] \\
& +\frac{t^{2}}{t^{2 \beta}(t-1)^{2}}\left(b_{11} b_{22}-b_{12} b_{21}\right)^{2} .
\end{aligned}
$$

For stability it would be sufficient that $|1+g(t, \beta)|<4$.

2. Proof of Theorem 1. Let $z_{i}(t)=e^{-\lambda t} x_{i}(t), i=1, \cdots, n$, where $\lambda$ is a fixed real number. Substituting into (1) we obtain the system 


$$
\begin{aligned}
z^{\prime}(t)= & \left(A_{0}(t)-\lambda I\right) z(t) \\
& +\sum_{l=1}^{m} A_{l}(t) \exp \left(-\lambda \tau_{l}(t)\right) z\left(t-\tau_{l}(t)\right), \quad t>t_{0},
\end{aligned}
$$

where $z(t)=\left(z_{1}(t), \cdots, z_{n}(t)\right)$. We will investigate the stability of the trivial solution of this system. To this end we take the Lyapunov functional

$$
\phi(t)=\|z(t)\|_{2}^{2}+\|z(t)\|_{\tau_{2}}^{2} .
$$

It is easily seen that this functional satisfies the conditions of the Krasovskif stability theorem [3]. Along the trajectories of $\left(1^{\prime}\right)$

$$
\begin{aligned}
-\frac{d \phi}{d t}= & \sum_{i=1}^{n} \sum_{j=1}^{n}\left\{(2 \lambda-m) \delta_{i j}-2 A_{0 i j}(t)\right\} z_{i}(t) z_{j}(t) \\
& +\sum_{i=1}^{n} \sum_{l=1}^{m}\left(1-\tau_{l}^{\prime}(t)\right) z_{i}^{2}\left(t-\tau_{l}(t)\right) \\
& -2 \sum_{i=1}^{n} \sum_{j=1}^{n} \sum_{l=1}^{m} A_{l i j}(t) \exp \left(-\lambda \tau_{l}(t)\right) z_{i}(t) z_{j}\left(t-\tau_{l}(t)\right) .
\end{aligned}
$$

For fixed real $\lambda$, the trivial solution of $\left(1^{\prime}\right)$ is stable if the quadratic form in the $n(m+1)$ variables $z_{i}(t), z_{i}\left(t-\tau_{l}\right), i=1, \cdots, n$, $l=1, \cdots, m$, is positive definite for all $t>t_{0}$. It is not difficult to see that this will indeed be so for sufficiently large positive values of $\lambda$. Indeed, the diagonal terms can be made as large as we please, whereas the off diagonal terms approach zero as $\lambda$ becomes large. The matrix associated with the quadratic form (4) can be written in block form as

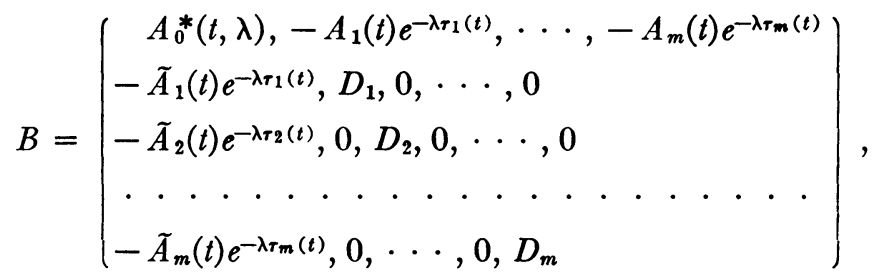

where

$$
\begin{aligned}
A_{0}^{*}(t, \lambda) & =(2 \lambda-m) I_{n}-\left(A_{0 i j}(t)+A_{0 j \boldsymbol{i}}(t)\right), \\
D_{\boldsymbol{i}} & =\left(1-\tau_{\boldsymbol{i}}^{\prime}(t)\right) I_{n},
\end{aligned}
$$

and $I_{n}$ is the unit $n \times n$ matrix.

The roots of $|B-\mu I|=0$ shall be real and positive for fixed $t>t_{0}$ and sufficiently large $\lambda$. For sufficiently small $\lambda$ the quadratic form 
in (4) shall be nonpositive and since the roots of $|B-\mu I|=0$ are continuous functions of $\lambda$ at least one root shall be zero for some value of $\lambda[4]$. These values of $\lambda$ are the values of $\lambda$ satisfying $|B(\lambda, t)|$ $=0$. It is easily seen [4] that

$$
|B(\lambda, t)|=\prod_{i=1}^{m}\left(1-\tau_{i}^{\prime}(t)\right) D(\lambda, t),
$$

where $D(\lambda, t)$ is defined by (2). Since by assumption $1-\tau_{i}^{\prime}(t) \neq 0$ all $i$ and $t,|B(\lambda, t)|=0$ implies $D(\lambda, t)=0$. Let $M(t)$ denote the largest of the real roots of $D(\lambda, t)=0$. Suppose $M(t) \leqq \gamma<0$ and take $\gamma<\lambda_{0}<0$. Then the quadratic form in (4) shall be positive definite for all $t>t_{0}$ if $\lambda=\lambda_{0}$. But this implies that $z_{i}(t)=\exp \left(\left|\lambda_{0}\right| t\right) x_{i}(t) \rightarrow 0$ as $t \rightarrow \infty$, $i=1, \cdots, n$. Thus $\|x(t)\| \leqq k \exp \left(-\left|\lambda_{0}\right| t\right)$ for some $k>0$. This proves the asymptotic stability of the trivial solution of (1).

REMARK. For stability of the trivial solution of (1) it is sufficient that all the roots $\mu(\lambda, t)$ of the polynomial $|B(\lambda, t)-\mu(\lambda, t) I|=0$ be positive for some $\lambda<0$ uniformly in $t$. This would be a more general, albeit, more cumbersome, criterion of stability than that of Theorem 1 .

\section{REFERENCES}

1. R. E. Bellman and K. L. Cooke, Differential-difference equations, Academic Press, New York, 1963. MR 26 \#5259; errata MR 27 p. 1399.

2. L. E. El'sgol'ts, Introduction to the theory of differential equations with deviating arguments, "Nauka," Moscow, 1964; English transl., Holden-Day, San Francisco, Calif., 1966. MR $30 \# 290$; MR 33 \#381.

3. N. N. Krasovskir, Stability of motion; applications of Lyapunov's second method to differential systems and equation with delay, Fizmatgiz, Moscow, 1959; English transl., Stanford Univ. Press, Stanford, Calit., 1963. MR 21 \#5047; MR 26 \#5258.

4. W. Hahn, Theory and application of Lyapunov's direct method, Ergebnisse der Mathematik und ihrer Grenzgebiete, Heft 22, Springer-Verlag, Berlin and New York, 1959; English transl., Prentice-Hall, Englewood Cliffs, N. J., 1963. MR 21 \#5048; MR 26 \#5230.

5. M. Oğuztöreli, Time-lag control systems, Mathematics in Science and Engineering, no. 24, Academic Press, New York, 1966. MR 36 \#484.

6. F. R. Gantmacher, Theory of matrices, GITTL, Moscow, 1953; English transl., Vols. 1, 2, Chelsea, New York, 1959. MR 16, 438; MR 21 \#6372c.

Wayne State University, Detroit, Michigan 48202 